\title{
Tuberculosis extra-pulmonar en niños bajo 15 años de edad internados en el Centro Hospitalario Pereira Rossell, Uruguay
}

\author{
Extrapulmonary tuberculosis in children under the age of 15 hospitalized at the Pereira Rossell Hospital \\ Center, Uruguay
}

Lucía Martínez', Silvina Vázquez', María de los Milagros Flores¹, Martin Notejane², Cristina Zabala², Gustavo Giachetto², Gabriela Sisto³ y Patricia Barrios ${ }^{2}$

\begin{abstract}
${ }^{1}$ Facultad de Medicina. Universidad de la República. Montevideo, Uruguay.
${ }^{2}$ Departamento de Pediatría. Facultad de Medicina. Universidad de la República. Montevideo, Uruguay.

${ }^{3}$ Comisión Honoraria para la Lucha Antituberculosa y Enfermedades Prevalentes. Uruguay.
\end{abstract}

Los autores declaran no tener conflictos de interés.

No hubo financiamiento externo.

Recibido: 31 de mayo de 2020 / Aceptado: 10 de septiembre de 2020

\section{Resumen}

Introducción: La tuberculosis es un problema de salud pública de alta prevalencia. Los niños constituyen una población de riesgo de enfermar y evolucionar a formas graves. Objetivo: Describir la frecuencia, características epidemiológicas, clínicas y evolutivas de los pacientes bajo 15 años de edad, internados por tuberculosis extra-pulmonar (TBCEP) en el Centro Hospitalario Pereira RossellUruguay, durante 2009 a 2019. Pacientes y Métodos: Estudio descriptivo, retrospectivo, revisión de historias clínicas y registros de la Comisión Honoraria para la Lucha Antituberculosa. Variables: edad, sexo, nexo epidemiológico, clínica, confirmación diagnóstica, tratamiento y complicaciones. Resultados: Se registraron 77 casos de TBCEP, fueron hospitalizados en este centro 45 (58\%). Media de edad 7 años, varones 25 (56\%). Todos recibieron vacuna BCG. Se identificó nexo epidemiológico en $28(62 \%)$. Las formas de TBCEP fueron: pleural 26 (58\%), sistema nervioso central (SNC) $9(20 \%)$, linfoganglionar $4(9 \%)$, cutánea $2(5 \%)$, ósea $1(2 \%)$, peritoneal $1(2 \%)$, pleural-peritoneal 1 (2\%), ósea-SNC 1 (2\%). Confirmación etiológica hubo en 23 (51\%): mediante cultivo 16, GeneXpert 5, por ambos 2. Completaron tratamiento $36(80 \%)$. Presentaron complicaciones 4 (9\%): status convulsivo 2, hemorragia cerebral 1, fallo orgánico múltiple 1. Conclusiones: La TBCEP ocurrió en niños previamente sanos. El diagnóstico requiere alto índice de sospecha y la confirmación el empleo simultáneo de varias técnicas diagnósticas.

Palabras clave: tuberculosis; Mycobacterium tuberculosis; niños; vacuna BCG; diagnóstico.

\begin{abstract}
Background: Tuberculosis is a public health problem. Children constitute a population at risk of becoming ill and evolving into serious forms. Aim: To describe the frequency, epidemiological, clinical and evolutionary characteristics of children under 15 years of age hospitalized for extrapulmonary tuberculosis (EPTB) at the Pereira Rossell Hospital-Uruguay, during 2009-2019. Methods: Descriptive, retrospective study, review of medical records and records of the Honorary Commission for the Fight Against Tuberculosis. Variables: age, sex, epidemiological and clinical link, diagnostic confirmation, treatment and complications. Results: 77 cases of EPTB were registered, $45(58 \%)$ were hospitalized in this center. Average age 7 years, males 25 (56\%). All received the Bacillus Calmette-Guérin vaccine. An epidemiological link was identified in $28(62 \%)$. The forms of EPTB were: pleural 26 (58\%), central nervous system (CNS) $9(20 \%)$, lymphoganglionic $4(9 \%)$, cutaneous $2(5 \%)$, bone $1(2 \%)$, peritoneal $1(2 \%)$, pleural- peritoneal $1(2 \%)$, bone-CNS $1(2 \%)$. Etiology was confirmed in 23 (51\%): by culture 16, GeneXpert 5, by both 2 . Thirty-six (80\%) patients completed treatment. Four $(9 \%)$ presented complications: convulsive state 2, cerebral hemorrhage 1, multiple organ failure 1. Conclusions: EPTB occurred in previously healthy children. The diagnosis requires a high index of suspicion and the confirmation of the simultaneous use of several diagnostic techniques.

Keywords: tuberculosis; Mycobacterium tuberculosis; children; Bacillus Calmette-Guérin vaccine; diagnosis.
\end{abstract}




\section{Introducción}

L a tuberculosis (TBC) es una enfermedad reemergente y una causa importante de mortalidad. En 2018, 1,5 millón de personas murieron por esta enfermedad en el mundo, de éstos 250.000 fueron pacientes bajo 15 años de edad ${ }^{1}$. En Uruguay, en el mismo año, la tasa de incidencia de TBC en población general fue $28,6 / 100.000$, se confirmaron 1.002 casos, de los cuales 47 correspondieron a pacientes bajo 15 años de edad $(6,67 / 100.000)^{2}$.

Los niños constituyen una población vulnerable; tras la primoinfección presentan mayor riesgo de desarrollar la enfermedad y evolucionar a formas graves. Se estima que $10 \%$ de los niños infectados por Mycobacterium tuberculosis desarrollará la enfermedad tuberculosa (ET), la mayoría durante el año posterior a la primoinfección ${ }^{3}$. El riesgo de progresión de infección a enfermedad depende de numerosos factores, entre los que se destacan, además de la edad, el estado nutricional, la vacunación con bacilo Calmette-Guérin (BCG) y la presencia de inmunodeficiencias. Los niños infectados por virus de inmunodeficiencia humana (VIH) tienen 20 veces más probabilidades de desarrollar la enfermedad ${ }^{4}$.

Usualmente, los niños se infectan por contacto directo con adultos o adolescentes, generalmente familiares o del entorno cercano, por lo que constituyen casos centinelas. La confirmación de un caso de TBC infantil alerta sobre la infección en adultos cercanos. La identificación y tratamiento de los casos índices es un pilar fundamental para controlar la transmisión de la enfermedad ${ }^{5}$.

Aunque la TBC pulmonar es la forma de presentación más frecuente, tanto en adultos como en niños, la TBC extra-pulmonar (TBCEP) presenta mayor morbimortalidad $^{5,6}$. Estas formas de presentación incluyen TBC pleural, ganglionar, de sistema nervioso central (SNC), ósea, y menos frecuentemente TBC cutánea, genitourinaria y peritoneal ${ }^{4,6-11}$. En nuestro país, la presentación extrapulmonar más frecuente es la pleural ${ }^{5,9}$.

La TBCEP suele ser el resultado de la diseminación hematógena de la infección pulmonar y, por tanto, indica enfermedad de larga evolución, pudiendo ser un marcador de inmunodepresión. El diagnóstico de estas formas de presentación representa un desafío debido a las variadas manifestaciones clínicas, lo que exige un alto índice de sospecha. Estas formas paucibacilares requieren la aplicación de estrategias diagnósticas sistematizadas para evitar el retraso en el inicio del tratamiento y disminuir la morbimortalidad. La TBCEP en niños debería ser considerada una alerta en el control de la enfermedad, que traduce dificultades en la detección, abordaje oportuno y adecuado de los contactos. Es necesario contribuir a conocer la magnitud del problema para mejorar la calidad de las prácticas asistenciales.

\section{Objetivo}

Describir la frecuencia, características epidemiológicas, clínicas y evolutivas de los pacientes bajo 15 años de edad, internados por TBCEP en el Centro Hospitalario Pereira Rossell (CHPR).

\section{Pacientes y Métodos}

Se realizó un estudio observacional, descriptivo, retrospectivo, en el que se incluyeron todos los pacientes bajo 15 años de edad, internados por TBCEP en el CHPR entre el 1 de enero de 2009 y el 31 de diciembre de 2019.

Para la definición de casos se aplicaron las siguientes definiciones operativas:

Enfermedad tuberculosa: Prueba cutánea de derivado proteico purificado (PPD) positiva más síntomas y signos sugestivos y/o radiografías patológicas ${ }^{1,3,12}$.

TBCEP: ET que afecta órganos y tejidos fuera del parénquima pulmonar por diseminación hematógena y linfática ${ }^{7}$.

Enfermedad confirmada: Detección de M. tuberculosis (cultivo o biología molecular) en cualquier parte del organismo, secreciones, o productos patológicos originados a nivel de las lesiones?.

Se analizaron las siguientes variables: edad, sexo, estado nutricional, cobertura con BCG, co-morbilidades (desnutrición, inmunodeficiencias, enfermedades crónicas), topografía (pleural, ganglionar, SNC, ósea, peritoneal, otras), manifestaciones clínicas al ingreso o momento de la consulta, noción de contacto, realización de PPD, estudios diagnósticos, días de hospitalización, complicaciones, tratamientos (fármacos, cumplimiento; reacción adversa a medicamentos).

Se evaluó el estado nutricional considerando los valores de peso, talla e índice de masa corporal según las tablas de la Organización Mundial de la Salud (OMS).

Los puntos de corte para considerar PPD positivo fueron los recomendados por la literatura médica internacional ${ }^{13}$.

Se definió como radiografía de tórax patológica el hallazgo de al menos uno de los siguientes: consolidación parenquimatosa (bronconeumonía), efusión pleural, atelectasias, linfadenopatía intratorácica, patrón miliar, patrón intersticial, cavitaciones pulmonares ${ }^{14}$.

Para el diagnóstico de reacción adversa a medicamentos (RAM) se utilizó la definición propuesta por la Organización Mundial de la Salud (OMS) en el año 2002: "Reacción nociva y no deseada que se presenta tras la administración de un fármaco, a las dosis utiliza- 
das habitualmente en la especie humana, para prevenir, diagnosticar o tratar una enfermedad, o para modificar cualquier función biológica"1.

Los pacientes se captaron a través de la base de datos informatizada del CHPR y registros de la Comisión Honoraria para la Lucha Antituberculosa y Enfermedades Prevalentes (CHLA-EP). La fuente de datos fueron las historias clínicas y registros de la CHLA-EP. Se utilizó para la recolección de datos una ficha prediseñada.

\section{Análisis estadístico}

Las variables cualitativas y cuantitativas discretas se expresaron en frecuencias absolutas y relativas (\%), las variables cuantitativas continuas en medidas de tendencia central y su rango. Para procesar los datos se utilizó el programa estadístico informático SPSS versión 18.

\section{Aspectos éticos}

Los datos de los usuarios se manejaron con total anonimato, salvaguardando la confidencialidad y el secreto médico. Se contó con la aprobación de la dirección y del Comité de Ética en Investigación de la institución.

\section{Resultados}

Durante el periodo de estudio se registraron en Uruguay 477 casos de ET en niños bajo 15 años de edad, 77 de presentación extra-pulmonar. Egresaron del CHPR 45 niños con diagnóstico de TBCEP, tasa de egreso 0,38/100.000. La distribución por año de egreso se muestra en la Figura 1.

En la Tabla 1 se presentan las características clínicas de los niños hospitalizados por TBCEP. La media de edad fue 7 años (5 meses-14 años), varones 25 (56\%) y eutróficos 39 (87\%); presentaban co-morbilidades 16
(35\%) la mayoría de ellos asma. Todos habían recibido vacuna $B C G$.

Se identificó nexo epidemiológico con un adulto bacilífero en 28 casos (62\%), 26 familiares directos, un conviviente no familiar y un compañero de centro educativo.

Las formas de TBCEP diagnosticadas por orden decreciente de frecuencia fueron: pleural 26 (58\%), SNC nueve $(20 \%)$, ganglionar cuatro $(9 \%)$, cutánea dos $(5 \%)$, ósea una $(2 \%)$, peritoneal una $(2 \%)$, pleural-peritoneal una $(2 \%)$ y ósea-SNC una $(2 \%)$.

\begin{tabular}{|c|c|c|}
\hline Variable & Frecuencia absoluta & Frecuencia relativa (\%) \\
\hline \multicolumn{3}{|l|}{ Edad (años) } \\
\hline $0-2$ & 11 & 24 \\
\hline 3- 9 & 18 & 40 \\
\hline $10-15$ & 16 & 36 \\
\hline \multicolumn{3}{|l|}{ Sexo } \\
\hline Masculino & 25 & 56 \\
\hline Femenino & 20 & 44 \\
\hline \multicolumn{3}{|l|}{ Estado nutricional } \\
\hline Eutrófico & 39 & 87 \\
\hline Desnutrido & 6 & 13 \\
\hline Co-morbilidades* & 16 & 35 \\
\hline Asma & 11 & \\
\hline Desnutrición crónica & 6 & \\
\hline Prematuridad leve & 2 & \\
\hline Cobertura con BCG & 45 & 100 \\
\hline
\end{tabular}

*Se encontró la presencia de más de una co-morbilidad simultánea. TBCEP: tuberculosis extrapulmonar, CHPR: Centro Hospitalario Pereira Rossell.

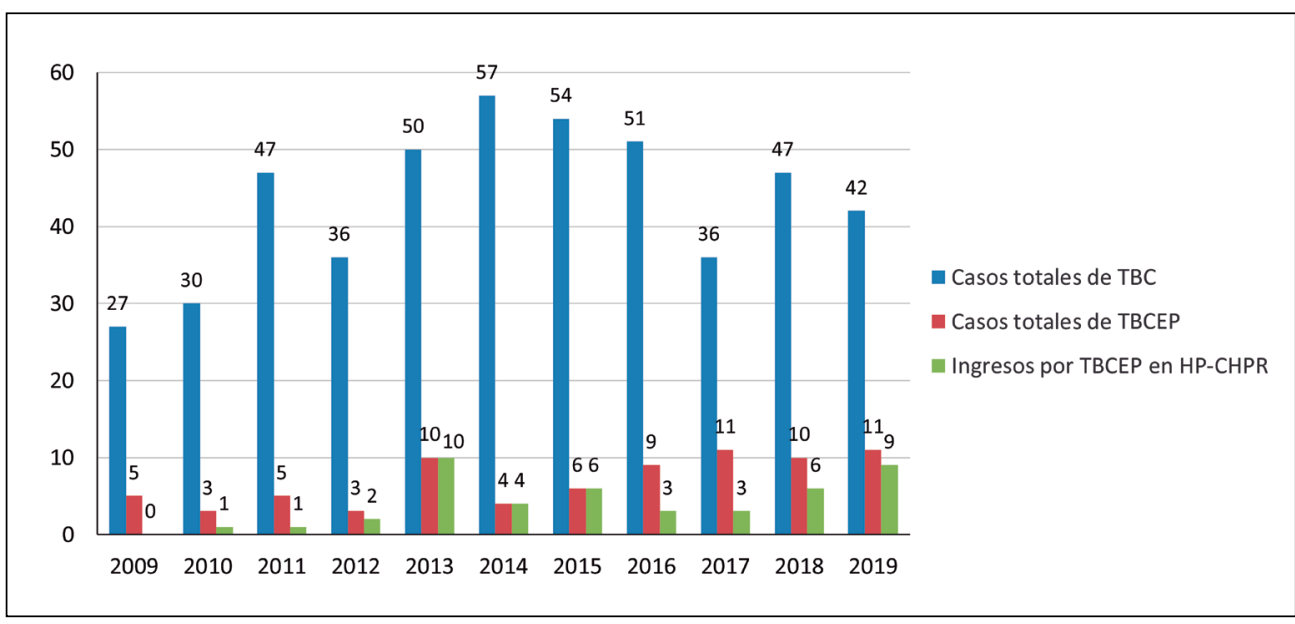

Figura 1. Distribución anual en Uruguay de casos de ET, TBCEP y egresos hospitalarios del CHPR por TBCEP en pacientes bajo 15 años de edad. Años 2009-2019. ET: enfermedad tuberculosa. TBCEP: tuberculosis extra-pulmonar. CHPR: Centro Hospitalario Pereira Rossell. 
Tabla 3. TBCEP en pacientes bajo 15 años de edad, internados en el CHPR, 2009-2019. Resultado de PPD y de los estudios microbiológicos (N: 45)

\begin{tabular}{lcc}
\hline Variable & Frecuencia absoluta & Frecuencia relativa (\%) \\
PPD positivo y forma de TBCEP & 29 & 64 \\
Pleural & 21 & 72 \\
Ganglionar & 3 & 10 \\
Sistema nervioso central & 2 & 7 \\
Cutánea & 1 & 3 \\
Ósea & 1 & 3 \\
Peritoneal & 1 & 3 \\
Confirmación diagnóstica & 23 & 51 \\
Baciloscopia (directo, cultivo) exclusivamente & 16 & 69 \\
Biopsia pleural & 7 & 44 \\
Tejido ganglionar & 5 & 31 \\
Líquido cefalorraquídeo & 2 & 13 \\
Lavado broncoalveolar & 1 & 6 \\
Tejido óseo & 1 & 6 \\
RPC- GeneXpert exclusivamente & 5 & 22 \\
Lavado broncoalveolar & 2 & 40 \\
Líquido pleural & 1 & 20 \\
Tejido óseo & 1 & 20 \\
Líquido cefalorraquídeo & 1 & 20 \\
Cultivo + RPC- GeneXpert & 2 & 9 \\
\hline
\end{tabular}

*Se registraron en dos casos la presentación de dos formas de tuberculosis extra-pulmonar (TBCEP) en forma simultánea. PPD: prueba cutánea de derivado proteico purificado. RPC: reacción de polimerasa en cadena.
Al momento de la consulta, la fiebre fue la manifestación más frecuente 29 (64\%) con una duración media de ocho días (1-30 días). En la Tabla 2 se muestra la topografía y manifestaciones clínicas.

Se realizó radiografía de tórax a todos los pacientes, fue patológica en 36 (80\%). Los hallazgos fueron: efusión pleural: 27, consolidación pulmonar: tres, infiltrado difuso: dos, patrón miliar: dos, atelectasia: una, adenopatías mediastinales: una. Los pacientes que presentaron radiografía normal fueron nueve, con TBC de SNC: cuatro, linfoganglionar: tres y cutánea: dos.

La adenosina deaminasa (ADA) en líquido pleural fue positiva en 21 casos de TBC pleural, la media de la concentración 77,52 UI/L (normal menor a $60 \mathrm{UI} / \mathrm{L}$ ).

Se realizó PPD en 38 de los 45 pacientes (84\%), fue positivo en 29 de ellos (76\%). En siete no fue posible realizarlo por carencia de reactivo.

A todos los niños se le solicitó estudio microbiológico (directo y cultivo) y RPC- GeneXpert a 25. (RPC: reacción de polimerasa en cadena) La TBCEP se confirmó en 23 niños (51\%), por cultivo 16, por RPC-GeneXpert cinco y en dos casos mediante cultivo y GeneXpert. En la Tabla 3 se muestran los resultados del PPD y estudios microbiológicos.

Tabla 2. TBCEP en pacientes bajo 15 años de edad, internados en el CHPR, 2009 - 2019. Topografía y manifestación clínica (N: 45)

\begin{tabular}{|c|c|c|c|c|c|c|c|c|c|}
\hline \multirow[b]{2}{*}{ Manifestación clínica } & \multicolumn{9}{|c|}{ Topografía o forma de presentación } \\
\hline & $\begin{array}{l}\text { Pleural } \\
(n=26)\end{array}$ & $\begin{array}{c}\text { SNC } \\
(n=9)\end{array}$ & $\begin{array}{l}\text { Linfogan- } \\
\text { glionar } \\
(n=4)\end{array}$ & $\begin{array}{l}\text { Cutánea } \\
(n=2)\end{array}$ & $\begin{array}{l}\text { Ósea } \\
(n=1)\end{array}$ & $\begin{array}{l}\text { Peritoneal } \\
\qquad(n=1)\end{array}$ & $\begin{array}{l}\text { Pleural- } \\
\text { peritoneal } \\
(n=1)\end{array}$ & $\begin{array}{l}\text { Ósea-SNC } \\
(n=1)\end{array}$ & Total \\
\hline Fiebre & 24 & 6 & 1 & 1 & 1 & 1 & 1 & 0 & 35 \\
\hline Tos & 22 & 4 & 1 & 0 & 1 & 0 & 0 & 0 & 28 \\
\hline Astenia & 6 & 0 & 0 & 0 & 1 & 1 & 0 & 0 & 8 \\
\hline Anorexia & 1 & 1 & 0 & 0 & 0 & 0 & 0 & 0 & 2 \\
\hline Adenopatías & 0 & 1 & 4 & 0 & 0 & 0 & 0 & 0 & 5 \\
\hline Sudoración nocturna & 7 & 1 & 0 & 0 & 1 & 1 & 0 & 0 & 10 \\
\hline Cefalea & 0 & 2 & 0 & 0 & 0 & 0 & 0 & 0 & 2 \\
\hline Vómitos & 5 & 4 & 0 & 0 & 0 & 0 & 1 & 0 & 10 \\
\hline Síndrome meníngeo & 0 & 1 & 0 & 0 & 0 & 0 & 0 & 0 & 1 \\
\hline Síndrome focal neurológico & 0 & 1 & 0 & 0 & 0 & 0 & 0 & 0 & 1 \\
\hline Dolor pleural & 5 & 0 & 0 & 0 & 0 & 0 & 0 & 0 & 5 \\
\hline Otros* & 3 & 3 & 1 & 2 & 1 & 0 & 1 & 1 & 12 \\
\hline
\end{tabular}

*Otros: dificultad respiratoria, alteración de conciencia, distensión abdominal, estado de mal convulsivo, gonalgia, deformidad ósea, eritema nodoso, cojera. TBCEP: tuberculosis extra-pulmonar. CHPR: Centro Hospitalario Pereira Rossell. SNC: sistema nervioso central. 
esta serie, la TBCEP se presentó en su mayoría en niños previamente sanos, sin co-morbilidades ${ }^{3,5,9,12,16-19}$.

La detección de un caso de TBC infantil constituye un evento centinela dado que indica infección reciente en adultos cercanos y su identificación constituye un pilar fundamental para el control de la transmisión ${ }^{5}$. En la mayoría de los niños incluidos en este estudio se logró detectar contacto con un caso índice, en general un adulto del núcleo familiar. La investigación de contactos debe ser amplia e incluir también familiares cercanos no convivientes y personas del entorno social incluyendo a centros educativos, como ocurrió en uno de los casos. El hallazgo de que en más de un tercio de los niños no se identificó el caso índice es un hecho preocupante ya que el diagnóstico precoz de los contactos resulta crucial en el control de la $\mathrm{TBC}^{3,5,20}$.

Al igual que lo descrito en otros estudios, dentro de las presentaciones de TBCEP, predominó la TBC pleural, seguida de SNC y linfoganglionar, siendo poco frecuentes la TBC cutánea, ósea y peritoneal ${ }^{6,7,21}$.

La sintomatología de presentación de TBCEP en niños y adolescentes está dada por una amplia variedad de manifestaciones clínicas, generalmente inespecíficas, lo que determina retraso en el diagnóstico, así como mayor morbimortalidad. Los síntomas más frecuentes fueron fiebre, tos y repercusión general, seguidos de múltiples síntomas, según la localización. Esto obliga al equipo de salud a realizar un correcto análisis epidemiológico y estar alertas ante la evolución atípica o no habitual de enfermedades prevalentes, como por ejemplo neumonía ${ }^{3,5,7,9,11,16,22}$.

El PPD es la prueba de elección para el cribado de esta enfermedad en la edad pediátrica. Permite detectar la exposición al bacilo, pero no distingue entre infección latente y enfermedad activa. En Uruguay, debido a que se realiza vacunación universal con BCG, se dificulta el análisis de este método de cribado. Por otra parte, se describe hasta $15 \%$ de falsos negativos vinculados a factores dependientes del hospedero (corta edad, infección reciente o diseminada, desnutrición, vacunaciones, tratamientos que produzcan inmunosupresión) y factores vinculados a la técnica. Algunos de estos factores podrían explicar los resultados falsos negativos observados en nueve niños ${ }^{5,9,16,23}$.

En la TBCEP, la confirmación diagnóstica representa un desafío. En estas formas de presentación, la baciloscopia y el cultivo presentan menor rendimiento debido a la naturaleza paucibacilar y las dificultades en la recolección de las muestras. Es importante recurrir a otras técnicas diagnósticas como la toma de biopsias con estudio anatomo-patológico y las técnicas de biología molecular como GeneXpert MTB/RIF. Esta última técnica, disponible en Uruguay desde el año 2014, presenta mayor sensibilidad que la baciloscopia, aunque ésta varía según las formas de presentación de la TBC siendo mayor en las formas 
óseas y linfoganglionares ${ }^{3,18,22-25}$. Un estudio realizado por Amaya y cols., evidenció mayor sensibilidad de GeneXpert MTB/RIF respecto a las baciloscopias, tanto para el diagnóstico de TBC pulmonar como TBCEP en niños ${ }^{27,28}$.

En esta serie se logró confirmación microbiológica en la mitad de los casos. La RPC- GeneXpert confirmó el diagnóstico en siete niños de nuestra casuística. La incorporación de esta técnica pudo haber contribuido al incremento del número de casos confirmados. Se destaca la necesidad de aplicar en forma simultánea varias técnicas de confirmación diagnóstica en la TBCEP.

Al igual que lo reportado en la literatura médica, en este estudio se constataron complicaciones en un número considerable de casos, siendo más frecuentes en las formas de TBC del SNC con presentación meníngea. Esto puede estar relacionado con retraso en el diagnóstico y tratamiento debido al bajo índice de sospecha de TBC en estas formas de variada presentación clínica ${ }^{9,14,29-32}$.

En 25 a $50 \%$ de los pacientes que sobreviven a la TBC del SNC se describen diversas secuelas: déficit intelectual, trastornos psiquiátricos, alteraciones sensoriales, epilepsia secundaria, hemiparesia y déficit motor, como se observó

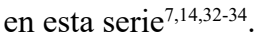

La mortalidad por TBCEP también se ve mayormente en las formas de presentación en el $\mathrm{SNC}$, en especial en la meningitis $(20$ a $40 \%)$ a pesar del tratamiento antituberculoso. En el presente estudio, los dos pacientes fallecidos eran pre-escolares de tres años, con formas de presentación diseminada (en un caso TBC del SNC y ósea y en el otro paciente, del SNC exclusivamente). En estos casos, la edad representa el factor de riesgo para la progresión rápida y mayor mortalidad ${ }^{16,22}$.

Un hallazgo importante fue el alto nivel de cumplimiento del tratamiento antituberculoso en los niños estudiados. El acceso al tratamiento, su administración supervisada y la facilitación del transporte a los centros de salud, son algunos de los factores que contribuyen a mantener altos grados de cumplimiento terapéutico.

La multi-resistencia a los fármacos antituberculosos constituye un problema creciente en el mundo. Si bien, en Uruguay se han registrado casos de multi-resistencia en población adulta, éste no ha sido un problema en la edad pediátrica ${ }^{5,29}$.

\section{Conclusiones}

La TBCEP ocurrió mayoritariamente en niños previamente sanos. Al igual que lo descrito con anterioridad por otros autores, las formas pleurales fueron las más frecuentes y las del SNC las de mayor morbimortalidad. Su diagnóstico oportuno requiere un alto índice de sospecha y su confirmación, el empleo simultáneo de varias técnicas diagnósticas. La identificación del caso índice continúa siendo el mayor desafío e implica la búsqueda activa de los posibles contactos convivientes y no convivientes.

\section{Referencias bibliográficas}

1.- WHO. Tuberculosis. Datos y cifras, 2019. Obtenido de: https://www.who.int/es/newsroom/fact-sheets/detail/tuberculosis. [consulta: 17 de enero de 2020].

2.- Comisión Honoraria para la lucha antituberculosa y enfermedades prevalente. Informe de la situación de la Tuberculosis en Uruguay, 2018. http://chlaep.org.uy/wpcontent/uploads/2019/05/Situaci\%C3\%B3nTuberculosis-Uruguay-2018.pdf. [consulta: 27 de febrero de 2020].

3.- Amaya G, Badía F, Pujadas M, Sisto G, Pírez C. Tuberculosis en la edad pediátrica. En: Guía Nacional para el manejo de la tuberculosis en situaciones especiales, año 2017. Montevideo, 2017. https://www. paho.org/uru/index.php?option=com docman\&view=download\&slug=guia-manejotuberculosis-2017-web\&Itemid=307. [consulta: 17 de febrero de 2020].

4.- Consejería de Sanidad, ed. INFORMES: Registro Regional de casos de tuberculosis de la Comunidad de Madrid. Informe del año 2016. Registro Regional de casos de tuberculosis de la Comunidad de Madrid.
Informe del año 2015. Boletín Epidemiológico de la Comunidad de Madrid 2017; 23 (7): 1-65. http://www.madrid.org/bvirtual/BVCM020176. pdf. [consulta: 17 de enero de 2020].

5.- Machado M, Pereira V, Coedo V, Arana M, Assandri E, Sisto G, et al. Características epidemiológicas y clínicas de niños hospitalizados por enfermedad tuberculosa: Centro Hospitalario Pereira Rossell (20102013). Rev Med Urug 2015; 31 (3): 172-8. http://www.scielo.edu.uy/pdf/rmu/v31n3/ v31n3a04.pdf.

6.- Chaves W, Buitrago J, Dueñas A, Bejarano J. Acerca de la tuberculosis extrapulmonar. Repert Med Cir 2017; 26 (2): 90-7. https:// www.sciencedirect.com/science/article/pii/ S0121737217300183.

7.- Ramírez-Lapausa M, Menéndez-Saldaña A, Noguerado-Asensio A. Tuberculosis extrapulmonar, una revisión. Rev Esp Sanid Penit 2015; 17: 3-11. http://scielo.isciii.es/pdf/ sanipe/v17n1/02_revision.pdf.

8.- $\quad$ Mellado M, García S, Baquero-Artigao F, Moreno D, Pineiro R, Méndez A, et al. Actualización del tratamiento de la tuberculosis en niños. An Pediatr (Barc) 2018; 88 (1): 52.e152.e12. https://www.analesdepediatria.org/ es-actualizacion-del-tratamiento-tuberculosisninos-articulo-S1695403317302254.

9.- Holcberg M, Zabala C, Gutiérrez S, Sisto G, Sosa M, Giachetto G. Prevalencia y características clínico-epidemiológicas de los niños con tuberculosis diagnosticados a partir de un caso índice. Uruguay 2012-2014. Arch Pediatr Urug 2016; 87 (S1): S3-S10. https://www.sup.org.uy/archivos-de-pediatria/ adp87s1/web/pdf/adp87s1_holcberg-tbc.pdf.

10.- Giachetto G. Tuberculosis en niños: una enfermedad reemergente. Arch Pediatr Urug 2013; 84 (3): 179-80. http://www.scielo.edu.uy/ pdf/adp/v84n3/v 84n3a01.pdf.

11.- García E, Yera D, Valdés S, Hernández M, Rives R. Comportamiento de la tuberculosis extrapulmonar en el Hospital Neumológico "Benéfico Jurídico" durante el quinquenio 1999-2003. Rev Cubana Med Trop 2006; 58 (3): 190-3. http://scielo.sld.cu/pdf/mtr/v58n3/ mtr03306.pdf.

12.- Machado K, Pereira V, Pírez C. Tuberculosis infantil: un caso clínico de presentación atípica. Arch Pediatr Urug 2015; 86 (1): 30-4. http:// www.scielo.edu.uy/pdf/adp/v86n1/v86n1a06. pdf.

13.- Highsmith H Y, Starke J R, Mandalakas A 
M. Tuberculosis. En: Wilmott R, Bush A, Deterding R, Ratjen F, Sly P, Zar H, et al. Kendig. Enfermedades respiratorias en niños. $9^{a}$ edición. Barcelona: Elsevier Health Sciences, 2019; 475-97.

14.- American Academy of Pediatrics. Tuberculosis. In: Kimberlin DW, Brady MT, Jackson MA, Long SS, eds. Red Book 2018. Report of the Committee of Infectious Diseases. 31st ed. Itasca Il. American Academy of Pediatrics; 2018; 829-53.

15.- Laporte J, Capellà D. Mecanismo de producción y diagnóstico clínico de los efectos indeseables producidos por medicamentos. En: Laporte J, Tognoni G. Principios de epidemiología del medicamento. $2^{\mathrm{a}}$ edición. Barcelona: Masson, 1993: 95-109.

16.- Moraes M, González S, García A, Menchaca A. Meningitis tuberculosa en un lactante. Reporte de caso y revisión de la literatura. Arch Pediatr Urug 2016; 87 (2): $137-$ 42. http://www.scielo.edu.uy/pdf/adp/v87n2/ v87n2a10.pdf.

17.- Carvalho A, Cardoso C, Martire T, Migliori G, Sant'Anna C. Epidemiological aspects, clinical manifestations, and prevention of pediatric tuberculosis from the perspective of the End TB Strategy. J Bras Pneumol 2018; 44 (2): 134-44. http:// www.scielo.br/pdf/jbpneu/v44n2/1806-3756jbpneu-44-02-00134.pdf.

18.- Cruz I, Salcedo M. Tuberculosis ganglionar. Experiencia en el Instituto Nacional de Enfermedades Respiratorias. Arch Pediatr Urug 2011; 82 (1): 18-22. http://www.scielo.edu.uy/ pdf/adp/v82n1/v82n1a04.pdf.

19.- Chiang S, Park S, White E, Friedman J, Cruz A, Del Castillo H, et al. Using changes in weight-for-age $Z$ score to predict effectiveness of childhood tuberculosis therapy. J Pediatric Infect Dis Soc 2020; 9(2): 150-8. https://doi.org/10.1093/jpids/ piy138.

20.- WHO. Tuberculosis. Estrategia Fin a la TB: objetivos e indicadores. https://www.who.int/ tb/strategy/end-tb/es/. (consulta: 17 enero de 2020).

21.- Du Preez K, du Plessis L, O'Connell N, Hesseling A. Burden, spectrum and outcomes of children with tuberculosis diagnosed at a district-level hospital in South Africa. Int J Tuberc Lung Dis. 2018; 22 (9): 1037-43. https://www.ingentaconnect.com/content/iuatld/ ijtld/2018/00000022/00000009/art00012.

22.- Casuriaga A, González K, Giachetto G, Costa F. Osteomielitis por Mycobacterium tuberculosis. Arch Pediatr Urug 2017; 88 (6): 322-8. http:// www.scielo.edu.uy/pdf/adp/v88n6/1688-1249adp-88-06-322.pdf.

23.- Bustamante J, Méndez-Echevarría A, Mellado M. Tuberculosis. En: Guerrero-Fernández J, Cartón A, Barreda A, Menéndez J, Ruíz J. Manual de Diagnóstico y Terapéutica en Pediatría. 6 a edición. Madrid: Panamericana, 2018: 1616-7.

24.- Méndez A, Mellado M, Baquero F, García M. Tuberculosis. En: Asociación Española de Pediatría. Sociedad Española de Infectología Pediátrica. Protocolos de Infectología. Madrid: Ergon, 2011: 103-12. https://www.aeped.es/ sites/default/files/documentos/tuberculosis.pdf. [consulta: 17 de enero de 2020].

25.- Budgell E, Evans D, Leuner R, Long L, Rossen S. The costs and outcomes of pediatric tuberculosis treatment at primary healthcare clinics in Johannesburg, South Africa. S Afr Med J. 2018; 108 (5): 423-31. http://www.samj. org.za/index.php/samj/article/view/12278/8464

26.- Gupta N, Kashyap B, Dewan P, Hyanki P, Singh N P. Clinical spectrum of pediatric tuberculosis: A microbiological correlation from a tertiary care center. J Trop Pediatr. 2018; 65 (2): 130-8. doi: 10.1093/tropej/fmy026.

27.- Amaya G, Contrera M, Arrieta F, Montano A, Pírez C. Rendimiento del GeneXpert en el diagnóstico de tuberculosis pulmonar y extrapulmonar en la edad pediátrica. Arch Pediatr Urug 2020; 91 (S2): S12-S23. https:// www.sup.org.uy/archivos-de-pediatria/ adp91-s2/web/amaya.html.
28.- Singh S, Singh A, Prajapati S, Kabra S, Lodha R, Mukherjee A, et al. Xpert MTB/RIF assay can be used on archived gastric aspirate and induced sputum samples for sensitive diagnosis of paediatric tuberculosis. BMC Microbiol 2015; 15: 191. https://bmcmicrobiol. biomedcentral.com/track/pdf/10.1186/s12866015-0528-z.

29.- Namsumba M, Kumkakumba E, Orikiriza P, Bastard M, Mwanga JA, Boum Y, et al. Treatment outcomes and tolerability of the revised WHO anti-tuberculosis drug dosages for children. Int J Tuberc Lung Dis. 2018; 22 (2): 151-7. https://www.ingentaconnect.com/ content/iuatld/ijtld/2018/00000022/00000002/ art00007\#.

30.- Jiménez Munguía R M, Huerta Romano J F. Tuberculosis. Formas clínicas extrapulmonares en los niños. Rev Mex Pediatr 2009; 76 (2): 8892. https://www.medigraphic.com/pdfs/pediat/ sp-2009/sp092g.pdf.

31.- Rosales Magallanes G. Meningitis tuberculosa en un lactante, a propósito de la aplicación de BCG a migrantes de zonas endémicas. Rev Mex Pediatr 2018; 85 (6): 222-5. https://www. medigraphic.com/pdfs/pediat/sp-2018/sp186f. pdf.

32.- Chiang S, Khan F, Milstein M B, Tolman A, Benedetti A, Starke J R, et al. Treatment outcomes of childhood tuberculous meningitis: a systematic review and meta-analysis. Lancet Infect Dis 2014; 14 (10): 947-57. doi: 10.1016/ S1473-3099(14)70852-7.

33.- Anderson N, Somaratne J, Mason D, Holland D, Thomas M. Neurological and systemic complications of tuberculous meningitis and its treatment at Auckland City Hospital, New Zealand. J Clin Neurosci. 2010; 17 (9): 1114-8. doi: 10.1016/j.jocn.2010.01.006.

34.- Miftode E, Dorneanu O, Leca D, Juganariu G, Teodor A, Hurmuzache M, et al. Tuberculous meningitis in children and adults: a 10-year retrospective comparative analysis. PLoS One 2015; 10 (7): e0133477. doi: 10.1371/journal. pone. 0133477. 